\title{
Exogenous BDNF and Chondroitinase ABC Consisted Biomimetic Microenvironment Regulates Survival, Migration and Differentiation of Human Neural Progenitor Cells Transplanted into a Rat Auditory Nerve
}

\author{
Ajay Kale ${ }^{1,2 *}$, Ekaterina Novozhilova1,2, Ulrica Englund-Johansson ${ }^{3}$, Samuel I. Stupp ${ }^{4}$, \\ Björn Palmgren1, Petri Olivius ${ }^{1,2,5}$ \\ ${ }^{1}$ Department of Clinical Sciences, Intervention and Technology, Karolinska Institutet, Karolinska University \\ Hospital, Stockholm, Sweden \\ ${ }^{2}$ Division of Otorhinolaryngology, Department of Clinical and Experimental Medicine, University of Linköping, \\ Linköping, Sweden \\ ${ }^{3}$ Department of Ophthalmology, Institution of Clinical Sciences in Lund, Lund University, Lund, Sweden \\ ${ }^{4}$ Department of Chemistry, Materials Science and Engineering, and Medicine and Institute for \\ BioNanotechnology in Medicine, Northwestern University, Chicago, USA \\ ${ }^{5}$ Division of Otorhinolaryngology, Linköping University Hospital, Linköping, Sweden \\ Email: ${ }^{*}$ ajay.kale@ki.se
}

Received 21 February 2014; revised 20 March 2014; accepted 18 April 2014

Copyright (C) 2014 by authors and Scientific Research Publishing Inc.

This work is licensed under the Creative Commons Attribution International License (CC BY).

http://creativecommons.org/licenses/by/4.0/

c) (i) Open Access

\begin{abstract}
Current putative regeneration oriented studies express possible role of stem cell based implantation strategy in the restoration of fundamental perception of hearing. The present work utilizes a rat auditory nerve (AN) directed transplantation of human neural progenitor cells (HNPCs) as a cell replacement therapy for impaired auditory function. Groups of $\beta$-bungarotoxin induced auditory function compromised female rats were used to transplant HNPCs in the nerve trunk. In the treatment groups, brain derived neurotrophic factor (BDNF), peptide amphiphile nanofiber bioactive gel (Bgel) and Chondroitinase $A B C$ (ChABC), a digestive enzyme that cleaves the core of chondroitin sulphate proteoglycans, were added along with HNPCs while the control groups were with PA inert gel (Igel) and devoid of ChABC. Six weeks post transplantation survival, migration, and

"Corresponding author.
\end{abstract}

How to cite this paper: Kale, A., et al. (2014) Exogenous BDNF and Chondroitinase ABC Consisted Biomimetic Microenvironment Regulates Survival, Migration and Differentiation of Human Neural Progenitor Cells Transplanted into a Rat Auditory Nerve. Neuroscience \& Medicine, 5, 86-100. http://dx.doi.org/10.4236/nm.2014.52012 
differentiation of HNPCs were studied and compared. The groups treated with BDNF and Bgel showed improved survival and differentiation of transplanted HNPCs while the ChABC treated group showed significant migration of HNPCs along the AN and elongation of neuronal fibers along the nerve towards the cochlear nucleus $(\mathrm{CN})$ which was characterized by immunocytochemical markers for human Nuclei (HuN), human mitochondria (HuM) and neuronal $\beta$-tubulin (Tuj1). These findings show that addition of BDNF and ChABC consisted Bgel environment facilitated HNPC survival, migration and differentiation along the transplanted rat AN towards the CN. This transplantation strategy provides unique experimental validation for futuristic role of cell based biomaterial consisted neurotrophic factor application in clinically transferable treatment of sensorineural hearing loss (SNHL) along with cochlear implants (CI).

\section{Keywords}

\section{Auditory Nerve, Brain Derived Neurotrophic Factor, Chondroitinase, Cochlear Nucleus, Human Neural Progenitor Cells, Transplantation, Transitional Zone, Sensorineural Hearing Loss}

\section{Introduction}

Hearing loss of sensory neural origin contributes to the most of deafness related clinical cases worldwide [1] [2]. Auditory sensory structures like hair cells (HC) and spiral ganglion neurons (SGN) are the primary important structures for hearing which are lost due to acoustic trauma, infectious reaction, effect of ototoxic drugs or aging process [3]. Neural prostheses like cochlear implant (CI) or brain stem implant (BSI) utilize the mechanism of electrical stimulation of primary auditory neurons of cochlea [4]-[7]. These implants have provided some degree of improvement in the hearing ability but the effects are variable in terms of speech recognition and language orientation. The auditory sensory hair cells and neurons also undergo progressive irreversible degeneration and this process eventually leads to deafness [8]. Other than the loss of these sensory structures, any damage to the auditory nerve also leads to a variable degree of hearing loss throughout the hearing process [9]. So to salvage these structures from irreversible damage or to regenerate these lost sensory structures, various strategies have been tried or are in experimental trials. Cell transplantation strategy is one of the alternatives which shows promise to counteract the irreversible loss of auditory hair cells, neurons or damage to the auditory nerve [10] [11]. A primary objective is to deliver the putative cells into the ear, usually inner ear with minimal changes in the internal physiological setting. Although the inner ear is a specialized complex structure which lies very deep and it is very difficult to reach a specific area within, it has been possible to implant these cells strategically in different areas of inner ear, organ of corti or auditory nerve as well [12].

Stem cells have created considerable interest in the scientific community to investigate their role in cell based treatment for deafness, because of their ability to restore or regenerate damaged cells and tissues in the human body [13] [14]. Since these cells are multi-potent or pluri-potent they can give rise to any cell type in the body and their cell expansion and specialization can be channelized with the internal cues like growth factors. So the intrinsic stem cells from the inner ear or stem cells from extrinsic source, which could be transplanted into the ear, are being looked at and being tried to regenerate hair cells and primary neurons to replace those that have been lost [15] [16]. Attempts have been made to implant stem cells in the inner ear or auditory nerve in the animal models with significant success [13] [17]. Since auditory neuronal degeneration to some degree occurs because of depletion of endogenous neurotrophic support, use of growth factors along with the stem cells has been relinquished for further expansion of these cells inside the body [18]. Thus stem cell implantation strategy, although in primitive stage, has been looked upon as the next promising therapy to restore hearing that could be in clinical use in near future. Taking into consideration the inadequate nature of endogenous regeneration ability or availability of the auditory precursor cells [19], some of the exogenous cell candidates and related approaches have been tried that could be beneficial in survival and differentiation of these implanted cells [20] [21]. Consequently, this will be helpful in the regeneration of sensory structures and could aid in treatment of the deafened auditory system.

Considering the fact that there is an irreversible loss of the sensory structures in the human ear, neural prosthetic devices have been developed and are being used as implants at the cochlear and brainstem levels to 
process the sound and convert it into electric signals for further processing in the higher brain loci [22]. The CI is surgically implanted and the electrodes stimulate the SGNs to further process the auditory signals to the brain, while the ABI has electrodes surgically implanted directly into the brain stem. The CI is mostly used to stimulate the SGNs in the cochlea while the ABI is used to stimulate the cochlear nucleus located in the brain stem. The CI is mostly favored in the cases where the auditory nerve function is intact while those who have auditory nerve damage would be benefited with an ABI directly stimulating the cochlear nucleus. The CI offers a quite effective speech recognition and discrete sound recognition ability in settled hearing conditions [23]. Most of the patients can hear well with CI but the maintenance of the hearing ability is dependent on the functional integrity and sustainability of the CI. On the other hand, the ABI is used when there is actual auditory nerve damage and deafness can be corrected to some respect with stimulation of auditory nucleus of the brain stem. The lack of reliability of consistent performance and inter patient variability of these implants is giving well defined hearing function for longer duration hinder their success as the lone treatment for deafness. Due to the drawbacks and inability of these implants, strategies of cell based regeneration of auditory sensory structures are making ways to the basic auditory research with the hope to make it to the clinical practice in near future.

Although treatment of neurodegenerative diseases with the help of stem cells is still in its primitive stage [24], cell based transplantation strategy provides a unique opportunity to study the regenerative aspect of degenerated nerve associated animal models. Traumatized or damaged auditory nerve related impaired hearing investigational cases provide such an interventional opportunity to assess the irreversible loss of sensory structures like the bipolar SGNs in the inner ear. Such loss of these sensory structures eventually leads to a complete loss of AN related hearing function in these patients [9] and this central nervous system (CNS) related functional loss cannot be corrected or recovered with the use of an external prosthetic device like CI [25]. The reason being that these implanted devices need intact sensory structures and a functional AN to conduct electrical signals generated through the sensory structures in the PNS and transmit them to cochlear nucleus in the CNS. Although the BSIs are being tried as a treatment for hearing impairment of central origin, the outcome has not been consistent and satisfactory for the patients [26]. This has led to an experimental thought process to find and implement a much improved and different approach that would have a better outcome in terms of hearing function. Therefore, regeneration of the lost sensory structures with the help of embryonic or progenitor stem cells has been tried as an experimental strategy to restore hearing function. We have also followed a similar path and have showed that the mouse tau-green fluorescent protein (GFP) embryonic stem cells (ESC) as well as HNPCs in in-vitro slice culture settings have shown ability to survive, differentiate and integrate towards CN [27] [28] when transplanted in-vivo into the auditory nerve of a deafened rat, they showed survival and migration into the CN along with differentiation [29]. This suggests that this implantation strategy can be utilized as a model to further investigate other cell candidates and their ability to restore hearing function.

To restore the hearing function these implanted cells need to have a good survival rate and also would need to differentiate or develop into well functioning auditory neuronal units to conduct electrical signals. Our previous studies involving mouse Tau-GFP cells showed promising results with these aspects [29]. Also, it has been shown previously that the human neural stem cells can be derived from the auditory nerve sensory structures like SGNs and can be cultured in vitro [30]. Therefore, we wanted to evaluate similar parameters of human originated stem cells in in-vivo transplantation to further strengthen our experimental strategy focusing towards clinical goal. We utilized HNPCs which have ability to develop into neurons or glial cells with the cue of proper trophic factors and show well regulated axonal and dendritic outgrowth. Various studies deriving these cells from embryonic or adult tissues have shown that these cells can be directed to give rise to cells having neuronal phenotype with the help of exogenous addition of different neurotrophic factors like brain derived neurotrophic factor (BDNF), glial cell derived neurotrophic factor (GDNF) and neurotrophin-3 (NT-3) etc. [31]. This fundamental development and maintenance of these cells have been tried and tested in number of neurodegenerative disease animal models and have demonstrated a great deal of success in forming the CNS cellular phenotypes and expressing related signaling characteristics [32]. The HNPCs that are used in the present study have been well described previously. We have previously established a rodent model of selective AN lesion [33] and have injected HNPCs along with a PA Bgel only or with BDNF added in the Bgel [29]. This Bgel is a nanofiber gel consisting of self-assembling PA molecules designed to present the laminin epitope isoleucine-lysine-valinealanine-valine (IKVAV) to the transplanted cells that promote neurite growth and this gel has also shown to inhibit astrocyte formation [34]. The mouse Tau-GFP ESCs were shown to benefit when added to these gels and differentiate into neurons. Furthermore, in previous studies it has been suggested that the transitional zone (TZ) 
along the AN works as a myelin-astrocyte barrier and prevents the migrating cells or regenerating nerve fibers to penetrate the oligodendrocytes protected CNS from the Shwann cells designated PNS for further electrical communication [35]-[37]. It has been demonstrated previously that ChABC is an enzyme that cleaves the core chondroitin sulfate proteoglycan (CSPG) and is a major component of extracellular matrix that helps permeabilize the $\mathrm{TZ}$ to facilitate the migration or penetration of nerve fibers towards the CNS [38] [39]. Furthermore, ChABC has also been shown to have ability to prevent glial scars and to enhance remyelination. So we added ChABC as one more component along with BDNF and Bgel to the transplanted HNPCs to evaluate the ability of these cells to survive, migrate, differentiate and make connections to the CNS. In the previous study, we allowed these transplanted HNPCs a survival time of three weeks, after which we evaluated the viability of these cells. Similarly, this study was allowed to carry on for a longer survival period of six weeks. This was done to evaluate the developmental ability of these cells, over a longer duration of survival, to regenerate functional sensory auditory structures and to further evaluate efficiency of these cells to grow neurites and make connections centrally by passing the TZ. This would contribute to the fundamental aspect of functionality of these regenerated auditory sensory structures aiding the ability of CI to perform better over a longer period preventing sensorineural hearing loss.

\section{Materials and Methods}

\subsection{Generation and Expansion of the Human Neural Precursor Cell Line}

The human neural precursor cell line used for this study was originally established by L. Wahlberg, A. Seiger, and colleagues at the Karolinska University Hospital (original work with the cell line is described in [40] and was kindly provided to us via Prof. A. Björklund (Dept. Exp. Med. Sci., Lund University, Sweden). The HNPC cell line was derived from a forebrain tissue of a 7-week old (post conception) human embryo. The HNPCs were cultured in DMEM-F12 medium (Invitrogen) supplemented with $2.0 \mathrm{mM}$ L-glutamine (Sigma), 0.6\% glucose (Sigma), N2 supplement (Invitrogen) and $2.0 \mu \mathrm{g} / \mathrm{ml}$ heparin (Sigma) and grown as free floating clusters (neurospheres). The growth factors, human basic fibloblast growth factor (hbFGF, $20 \mathrm{ng} / \mathrm{ml}$; R\&D Systems), human epidermal growth factor (hEGF, $20 \mathrm{ng} / \mathrm{ml}$; Invitrogen) and human leukemia inhibitory factor (hLIF, $20 \mathrm{ng} / \mathrm{ml}$; Sigma) were added every 3 - 5 days to the culture. The neurospheres were passaged by mechanical dissociation every 7 - 10 days and reseeded as single cells at a density of $1 \times 10^{5}$ cells/ml. Cells used in the study were passaged 7 - 11 times [41].

\subsection{Experimental Animals}

All animal experiments followed the national approved protocol for care and use of animals in Sweden (N3/11; N4/11). Young adult female Sprague Dawley rats (200 - 250 gm body weight) were housed in groups under a 14 hr light: $10 \mathrm{hr}$ dark lighting schedule and permitted free access to standard rat chow and water. Preoperative otoscopic examinations were performed to exclude any visible middle ear infection.

\subsection{Deafening of Animals}

All the animals were deafened by an application of $\beta$-bungarotoxin ( $\beta$-BuTx, Alexis Biochemicals) to the round window niche as described previously [33]. In brief, after i.p. (intra peritoneal) anesthesia with xylazine (10 $\mathrm{mg} / \mathrm{kg}$ i.p.) and ketamine (50 mg/kg i.p.) the round window niche was exposed by a retro auricular incision. $5 \mu \mathrm{l}$ of $\beta$-BuTx $(0.05 \mu \mathrm{g} / \mathrm{ml})$ was absorbed on a piece of gel foam and applied to completely fill the round window niche. A piece of fascia was placed to cover the hole in the bulla and the wound was stitched closed. The animals were allowed to recover for three weeks until further surgical procedures were performed.

\subsection{Cell Implantation}

The surgical approach for the injections to the auditory nerve trunk by the intraauditory meatus (IAM) (Figure 1) has been previously described [29]. In brief, all the animals were anaesthetized with an i.p. injection of a mixture of Ketalar@ (50 mg/kg) and Rompun $(5)(10 \mathrm{mg} / \mathrm{kg})$ and the skull was put in a fixed position with the aid of a stereotactic frame. Under a surgical microscope an incision was made through the skin and underlying soft tissue. Using a drill, a hole was made in the sub-occipital bone and the underlying dura was opened and reflected 
towards the edge of the hole. The cerebellar hemisphere was retracted medially to reveal the auditory nerve entering through the IAM. For injections, a 30-gauge needle (Hamilton, Switzerland) attached to a $10-\mu$ microsyringe (Hamilton, Switzerland) was mounted to the stereotactic frame. The needle was positioned above the auditory nerve trunk with an angle of the tip adjusted towards the IAM. The needle was then lowered into the auditory nerve trunk by the use of a micromanipulator. A total volume of $5 \mu \mathrm{l}$ containing the cells at a concentration of approximately $1 \times 10^{5}$ cells/ml in medium either alone or along with different adjuvants according to the treatment groups as depicted in Table 1, was injected into the nerve trunk over a period of 1 minute. After injection the needle was left in place for 10 minutes, and then the wound cavity was filled with sterile saline. A piece of fascia was used to cover the hole in the dura and occipital bone. The wound was sutured in layers and stitched closed. Following the surgery and removal from the stereotactic frame, the animals were given subcutaneous injections of $3 \mathrm{ml}$ saline for rehydration and $0.2 \mathrm{ml}$ Temgesic $@(0.3 \mathrm{mg} / \mathrm{ml})$ for pain relief and were placed in a warm cage to recover before being transferred to the home cage. To prevent postoperative infection and immune response rejection, all the animals received daily doses of tetracycline $(1.8 \mathrm{mg} / \mathrm{ml}$, i.p.) and cyclosporine $(4.2 \mathrm{mg} / \mathrm{ml}$, i.p.). Sham surgery was done consisting of medium injected by the IAM. In all the animals, cells were injected together with $5 \mu \mathrm{l}$ of culture medium containing supplementary BDNF $(0.001 \mathrm{mg} / \mathrm{ml})$. The treatment groups received additional BDNF $(10 \mathrm{mg} / \mathrm{ml})$, ChABC $(200 \mathrm{U} / \mathrm{ml})$ or BDNF/ChABC mixed in 1\% IKVAV peptide amphiphile (PA) nanofiber bioactive hydrogel (Sequence: C16-VVAAEEEEGIKVAV) or inert control VVIAK PA gel (Sequence: C16-VVAAEEEEGVVIAK) (Nanotope, USA) [34]. Also, $5 \mu$ of the $\mathrm{BDNF} / \mathrm{ChABC}$ saturated hydrogel was applied around the injection site.

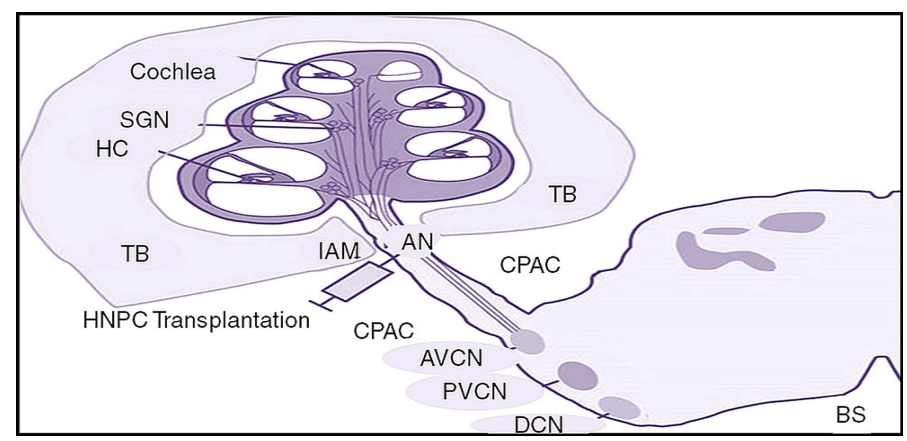

Figure 1. Schematic showing transplantation strategy in injecting HNPCs into a rat auditory nerve trunk through an IAM using a cerebro pontine angle. Abbreviations: AN, auditory nerve trunk; AVCN, anterior ventral cochlear nucleus; $\mathrm{BS}$, brain stem; CPAC, cerebro pontine angle; DCN, dorsal cochlear nucleus; HC, hair cells; IAM, internal auditory meatus; PVCN, posterior ventral cochlear nucleus; SGN, spiral ganglion neurons; TB, temporal bone.

Table 1. Treatment paradigm for the implantation of cells and modulatory factors into the rat AN. All the animals were deafened with $\beta$-bungarotoxin treatment prior to transplantation and then divided into 7 different treatment groups for transplantation of HNPCs into the rat AN. Along with these treatment groups sham operated control animals were devoid of HNPCs and received just the medium to the AN. The following combinations of modulatory factors were added along with HNPCs for the rat AN directed transplantation and also applied to transplantation site adjacent locations.

\begin{tabular}{|c|c|c|c|c|c|}
\hline Groups & Cells & Inert PA gel & Bioactive PA gel & BDNF & ChABC \\
\hline 1 & HNPC & - & - & - & - \\
\hline 2 & HNPC & + & - & - & - \\
\hline 3 & HNPC & - & + & - & - \\
\hline 4 & HNPC & + & - & + & - \\
\hline 5 & HNPC & - & + & + & - \\
\hline 6 & HNPC & + & - & + & + \\
\hline 7 & HNPC & - & + & + & + \\
\hline
\end{tabular}

BDNF, brain derived neurotrophic factor; ChABC, chondroitinase ABC; HNPC, human neural progenitor cells; PA, peptide amphiphile. 


\subsection{Immunofluorescence Histochemistry}

After 6 weeks of survival, all the rats were sacrificed by an overdose of pentobarbital $(60 \mathrm{mg} / \mathrm{ml}$, i.p.) and transcardially perfused with warm $0.9 \%$ saline followed by ice-cold $4 \%$ paraformaldehyde (PFA) in $0.1 \mathrm{M}$ phosphate buffered saline (PBS). The cochlea, the auditory nerve and the part of auditory BS were carefully removed en bloc. The specimens (cochlea plus auditory nerve including the brain stem) were dissected out and a small hole was used for PFA perfusion (initially $4 \%$ and then $0.5 \%$ ) into the cochlear apex. The cochlear specimens were decalcified in ethylene diamine tetraacetate (EDTA) for seven days. After decalcification, the specimens were placed in $20 \%$ sucrose solution for 24 hours and were embedded and frozen in OCT Compound (TissueTek; Sakura Finetek, Torrance, CA, USA). The specimens were oriented and cryosectioned using neuroanatomical landmarks depicted in "The rat brain in stereotaxic coordinates" (G. Paxinos and C. Watson, Elsevier 2005) as a guide. Consecutive $12 \mu \mathrm{m}$ thick sections were collected over approximate intervals of $-9.00 \mathrm{~mm}$ to -11.28 $\mathrm{mm}$ relative to bregma in order to obtain suitable mid-modiolar sections containing the cochlea, auditory nerve and the brain stem (BS) as a continuum. The sections were then mounted on glass slides and washed with $0.1 \mathrm{M}$ PBS. After stringent washing with PBS the sections were preincubated for 30 min with $5 \%$ bovine serum albumin (BSA) in PBS. The sections were subsequently incubated for $48 \mathrm{~h}$ at $4{ }^{\circ} \mathrm{C}$ with a cocktail consisting of rabbit polyclonal $\beta$-tubulin (Tuj1) (1:500; Covance Research Products, Berkeley, CA, USA) and mouse monoclonal human nuclei (HuN) (1:200; Millipore, Temecula, CA, USA) or mouse monoclonal human anti mitochondrial (HuM) (1:200, Millipore, Temecula, CA, USA) antibodies diluted in PBS containing $0.2 \%$ Triton X-100. Following incubation the samples were rinsed with PBS and incubated subsequently with goat-anti rabbit Cy3 (1:200) and Alexa Fluore 488 goat-anti mouse (1:500; Invitrogen) antibodies for 90 min each. Omission of the primary antibody served as negative control. Cell nuclei were stained with 4, 6-diamidino-2-phenylindole (DAPI). After subsequent washings with PBS, the sections were coverslipped using ProLong Gold antifade agent (Invitrogen). The specimens were visualized and photographed using a fluorescence laser scanning confocal microscope LSM 700 equipped with a digital camera (Zeiss). The images were acquired and processed using Zen 2010 software (Carl Zeiss, MicroImaging GmnH, Release version 6.0). For each animal, random blind replicate counts were performed at a magnification of $40 \times$. The number of implanted cells was defined as the number of cell profiles with co-expression of HuN and DAPI. For unbiased quantifications, a random starting section was selected from the 20 - 30 sections from each specimen. The cellular profiles were quantified in every third section until a total of nine sections were reached, ideally giving at least 100 profiles quantified for each specimen. Since the average diameter of the profiles quantified was $15 \mu \mathrm{m}$ this procedure avoided double counting of profiles. During the quantification process each and every analyzed section was subdivided into small fields out of which a selection of fields were analyzed in a regular fashion, each field being equivalent to the optic field in the microscope. The number of cells in each field was multiplied by the total number of fields in the evaluated section. In order to get an estimate of the total number of cells, the number of cellular profiles counted was multiplied by nine. HuN-immunopositive cells were defined by filling of nuclear compartment or HuM-immunopositive cells were defined by coverage of axonal or dendritic mitochondrial transport respectively by Alex Fluor 488 emitted light while Tuj1-immunopositive cells were characterized by Cy3 emitted light; duel labeled cells were characterized by superimposition of Alex Fluor 488 and Cy3 emissions labeling respective antigens.

\section{Statistical Analysis}

All the results were expressed as the mean \pm the standard error of the mean (SEM). The mean values were compared between the treatment groups by one-way ANOVA followed by Tukey's HSD test. Differences of $\mathrm{p}<$ 0.05 were considered significant.

\section{Results}

\subsection{Survival and Location of the Implanted HNPC Cells}

The data in Figure 2 show that mean numbers of survived HuN-ir-positive cells were not different between the control groups injected with HNPCs only versus HNPCs along with inert gel [HNPC + Igel] in any auditory sensory structural area evaluated 6 weeks after transplantation of HNPCs. The BDNF treatment in inert gel $[$ HNPC + Igel + BDNF] increased mean counts of HuN-immunolabeled cells in the AN, while BDNF along 


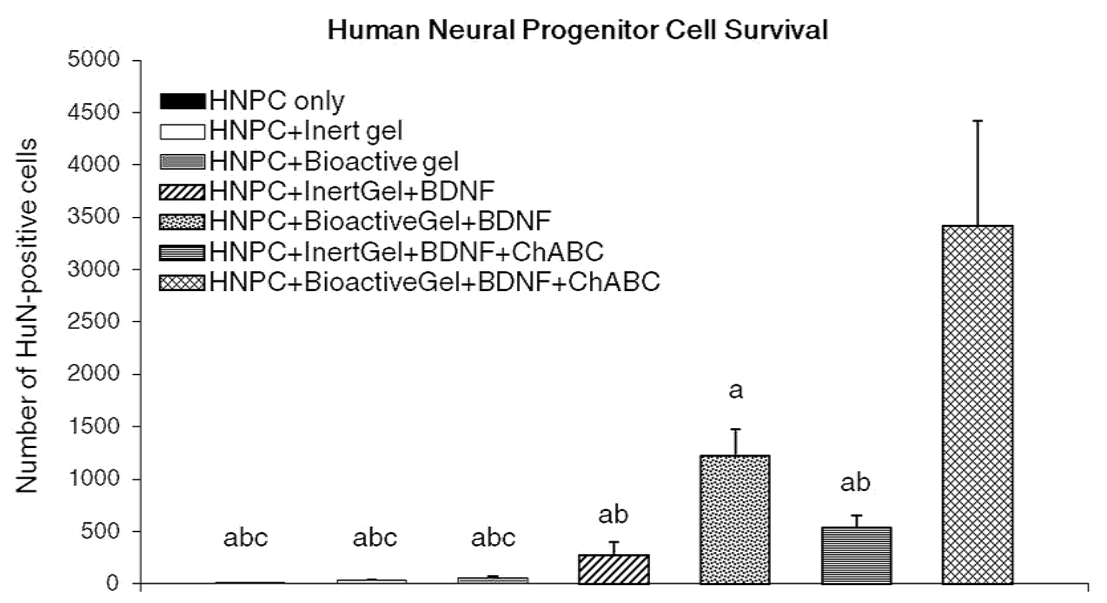

Figure 2. Effects of BDNF and ChABC treatment in the presence or absence of bioactive PA gel on the mean survival numbers of HuN-immunopositive HNPCs in characterized auditory loci in the female rats. $n=5$, Significant differences between treatments: ${ }^{a} P<0.05$, compared to HNPC + BioactiveGel + BDNF + ChABC; ${ }^{b} P<$ 0.05 , compared to HNPC + BioactiveGel + BDNF; ${ }^{c} P<0.05$, HNPC + InertGel + BDNF + ChABC. Abbreviations: BDNF, brain derived neurotrophic factor; Bgel, bioactive peptide amphiphile gel; ChABC, chondroitinase ABC; HNPC, human neural progenitor cells; Igel, inert control peptide amphiphile gel.

with bioactive gel [HNPC + Bgel + BDNF] significantly increased mean counts of HuN-immunolabeled cells along AN proximal to CN and into the CN areas, relative to the HNPC only and HNPC + Igel controls. Following addition of ChABC in inert gel [HNPC + Igel + BDNF + ChABC] numbers of HuN-containing cells in the AN proximal to CN were comparably increased similar to the [HNPC + Igel + BDNF] group while ChABC along with bioactive gel and BDNF [HNPC + Igel + BDNF + ChABC] treatment group showed marked increase in numbers of HuN-containing cells in the AN proximal to $\mathrm{CN}$ area as well as into the $\mathrm{CN}$ as compared to all the control and other treatment groups. The data show that, as the treatment accompanied the biomimetic factors there was significantly higher cell survival observed 6 weeks post transplantation. Also the cells showed complete migration from the site of transplantation towards the BS and the location of the cells was seen mainly along the $\mathrm{AN}$ area in proximity to the $\mathrm{CN}$ or converging towards the $\mathrm{CN}$ and in the $\mathrm{CN}$ which can be seen from Figure 3.

\subsection{Differentiation of Survived HNPCs}

Figure 3 illustrates representative patterns of staining for nuclear HuN-ir-positive cells, Tuj1-ir-positive neuronal cells and colocalization of HuN and Tuj1 in the AN area proximal to $\mathrm{CN}$ and into the $\mathrm{CN}$ for the treatment group HNPC + Bgel + BDNF after 6 weeks of survival period. There was significant increase in the number of surviving HNPCs along with their Tuj1 positive neuronal immunoreactivity as compared to the HNPCs only and HNPC + Igel control groups as well as HNPC + Igel + BDNF group. The surviving cells showed a greater population in $\mathrm{AN}$ area in close proximity to $\mathrm{CN}$ moving towards the $\mathrm{CN}$, along with the characteristics of neuronal phenotype showing differentiation.

Figure 4 shows that the mean numbers of survived HuN-ir-positive cells colocalized with Tuj1-ir-positive neuronal structures were not different between control groups injected with HNPCs only versus HNPCs along with inert gel [HNPC + Igel] in any auditory sensory structural area evaluated 6 weeks after transplantation of HNPCs. The BDNF treatment [HNPC + Igel + BDNF] significantly increased mean counts $(50 \%$ of total survived) of HuN and Tuj1-ir-coimmunolabeled cells in the AN while bioactive gel along with BDNF [HNPC + Bgel + BDNF] increased mean counts (60\% of total survived) of HuN and Tuj1-coimmunolabeled cells in the AN and CN, relative to the HNPC only and HNPC + Igel controls. Following addition of ChABC [HNPC + Igel $+\mathrm{BDNF}+\mathrm{ChABC}$ ] numbers of HuN and Tuj1 immmunolabel containing cells in the AN were comparably increased (45\% of total survived) similar to the [HNPC + Igel + BDNF] group while [HNPC + Igel + BDNF + $\mathrm{ChABC}$ ] treatment group showed marked increase in numbers (71\% of total survived) of HuN-Tuj1 colocalized 


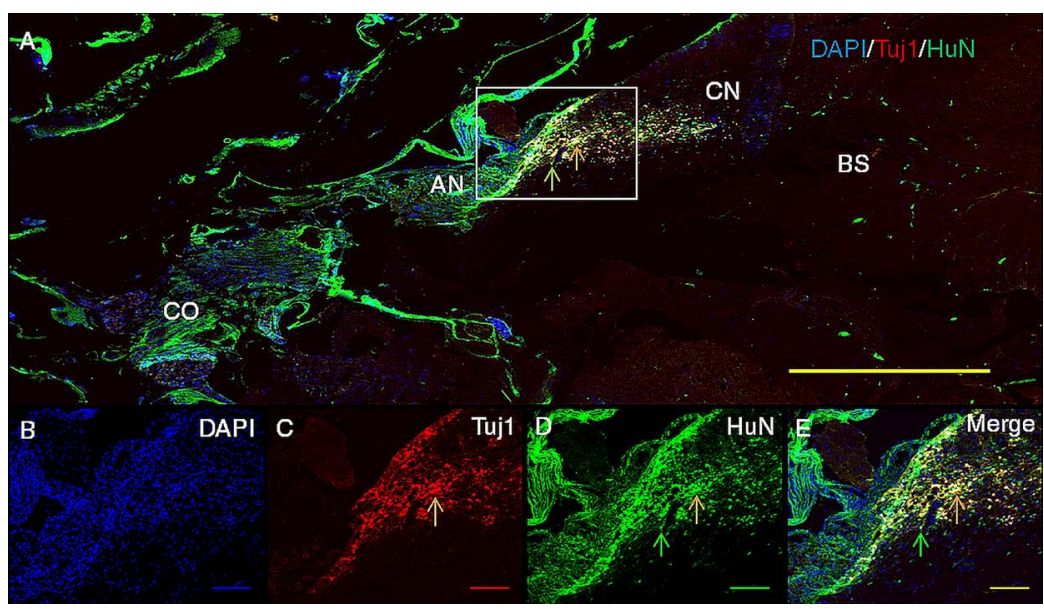

Figure 3. Representative macroscopic histological images showing survival and migration of the regenerated structures towards $\mathrm{CN}, 6$ weeks post transplantation of HNPCs along with BDNF and Bgel into the rat AN. Upper panels depict representative patterns of immunostaining for Tuj1 ir-positive HNPCs along the AN and CN (A, panoramic view, at 5× magnification with scale bar of $400 \mu \mathrm{m}$ (yellow) and lower panels depict representative patterns of immunostaining at $10 \times$ magnification with respective colored scale bars of $100 \mu \mathrm{m}$ for DAPI nuclear staining (B) (blue), Tuj1 neuronal staining (C) (red), HuN nuclear staining (D) (green), DAPI + Tuj1 + HuN co-expression (E) (yellow). Rectangle (white) indicates selected area of illustration. Arrows indicate that HNPCs are colabeled for Tuj1and HuN (orange) and single labeled for HuN alone (green). Abbreviations: AN, auditory nerve; BS, brain stem; CN, cochlear nucleus; $\mathrm{CO}$, cochlea.

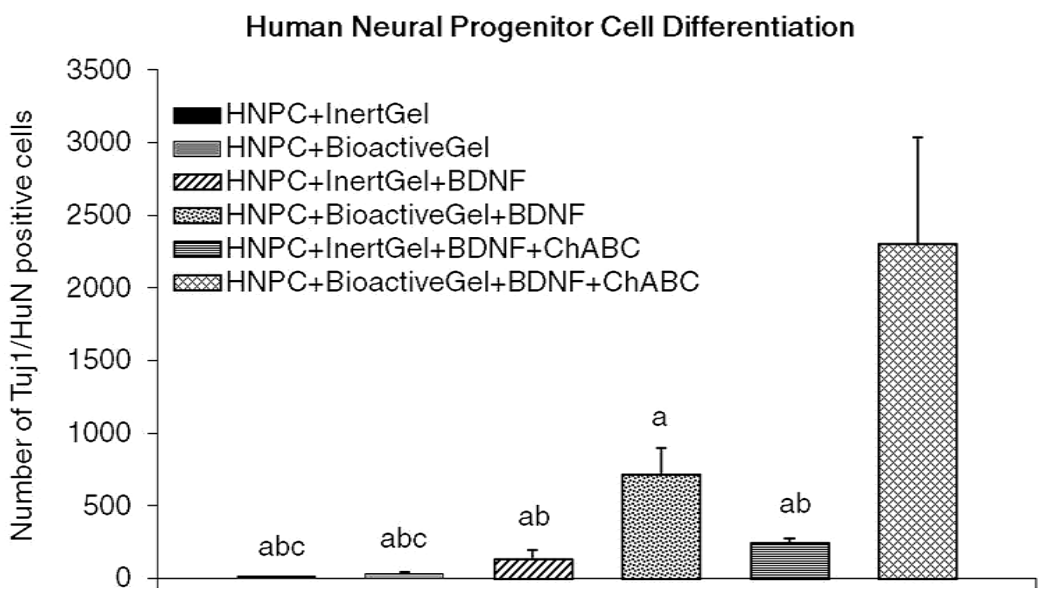

Figure 4. Effects of BDNF and ChABC treatment in the presence or absence of bioactive PA gel on the mean numbers of differentiated HuN-plus Tuj1 ir-positive HNPC derived neuronal structures in characterized auditory loci in the female rats. $\mathrm{n}=5$, Significant differences between treatments: ${ }^{a} P<0.05$, compared to HNPC + BioactiveGel + BDNF + ChABC; ${ }^{b} P<0.05$, compared to HNPC + BioactiveGel + BDNF; ${ }^{c} P<0.05$, HNPC + InertGel + BDNF + ChABC. Abbreviations: BDNF, brain derived neurotrophic factor; Bgel, bioactive peptide amphiphile gel; ChABC, chondroitinase ABC; HNPC, human neural progenitor cells; Igel, inert control peptide amphiphile gel.

neuronal cells in the AN as well as CN as compared to all the control and other treatment groups. The data show that the development of these cells towards a neuronal lineage with characteristic differentiation of the processes thereafter was seen in more numbers when the cells were implanted accompanied with the external modulatory internal physiological milieu mimicking factors. 
Figure 5 portrays representative patterns of staining for nuclear HuN-ir-positive cells, Tuj1-ir-positive neuronal cells and colocalization of HuN and Tuj1 in the AN and CN for the treatment group HNPC + Bgel + BDNF + ChABC after 6 weeks of survival period. ChABC treatment significantly increased number of surviving HNPCs and also duel immunolabeling for HuN and Tuj1 as compared to HNPCs only and HNPC + Igel control groups and other treatment groups. Significantly large number of surviving cells was observed in AN area proximal to $\mathrm{CN}$ and moving into the $\mathrm{CN}$ showing neuronal phenotypic characteristics along with differentiation.

\subsection{Sprouting of Axonal or Dendritic Extensions}

Data in the Figure 6, show that the mean numbers of Tuj-1-ir-positive and HuM-ir-positive neuronal structures showing sprouting in terms of axonal or dendritic extensions from the soma between the treatment groups were not different between inert gel treated BDNF group [HNPC + Igel + BDNF] and ChABC added group [HNPC + Igel $+\mathrm{BDNF}+\mathrm{ChABC}$ ] in any auditory sensory structural area evaluated 6 weeks after transplantation of HNPCs. The number of neurons showing such sprouting characteristics was significantly higher in the BDNF treatment group having the bioactive gel [HNPC + Bgel + BDNF] as compared to the other inert gel groups, while the ChABC added bioactive gel group [HNPC + Bgel + BDNF + ChABC] showed significantly high numbers of sprouting neurons with bi-to-multi polar extensions. The data show that there was appreciable number of neuronal cells that developed 6 weeks post transplantation to send out axonal or dendritic extensions along the $\mathrm{AN}$ in proximity to $\mathrm{CN}$ and also in to the CN. The multidirectional orientation of these neuronal processes was seen connecting or growing towards the BS as well.

The data in Figure 6 also depicts representative immunolabeling showing surviving HNPCs developing into neurons (Tuj1 positive) and extending axonal or dendritic outgrowths (HuM positive) in the Bgel + BDNF + ChABC cofactor added group after 6 weeks. The ChABC treatment showed higher neuronal differentiation and sprouting in CN area. Significantly higher bipolar to multi polar extensions were observed into the cochlear nucleus area. Some of the differentiated neuronal structures also showed branching, sending out processes towards the BS.

\section{Discussion}

The current study provides a novel evidence that HNPCs when transplanted along with BDNF, ChABC in PA bioactive gel shows survival over a longer period, migration through the transitional zone along the AN and that the magnitude of this cellular viability is further enhanced in a subset of these differentiating cells by axonal sprouting trying to make connections to the CNS. The data also demonstrates that ChABC provides a unique advantage to this implantation strategy and thereby HNPCs could permeate through the TZ. The present evidence that after six weeks of transplantation the HNPCs survived, migrated and differentiated in vivo supports the view that cell based transplantation strategy can be looked on as futuristic supplemental treatment along with hearing aids in hearing compromised cases.

The data obtained here indicates that 6 weeks after transplantation HNPCs showed relatively good number of cell survival amongst the BDNF and ChABC included treatment groups. Moreover, the extent of differentiation after the migration was seen to be better in ChABC + BDNF + Bgel treatment group as compared to all other non ChABC treated groups. Also, the axonal sprouting and elongation towards the BS was seen to be of higher degree in ChABC + BDNF + Bgel treated group. Our previous studies show that 3 weeks after transplantation there was appreciable increase in survival number of GFP-HNPCs and mouse tau-GFP cells showed a greater degree of survival, migration and differentiation when ChABC was added to the transplanted cells [29]. Those findings suggest that there exists a role of ChABC to influence cellular migration, cellular growth into neural and further axonal development. Rather, the present evidence of axonal or dendritic sprouting and characteristic bipolar neuronal resemblance in subset of cellular structures supports the view that ChABC has indeed upregulated the axonal growth activity by preventing or decreasing the formation chondroitin sulphate molecule. At present, the functional significance of these survived HNPCs in genomic regulatory function as functional auditory neurons remains unclear, but the ability of these progenitor cells to make these types of cells may be related to the appropriate local microenvironment modulation.

As previously established in various studies, key trophic factors like BDNF have shown to have favorable developmental effects on the auditory SGNs [42]. SGNs are primarily well supported by BDNF secreted from 


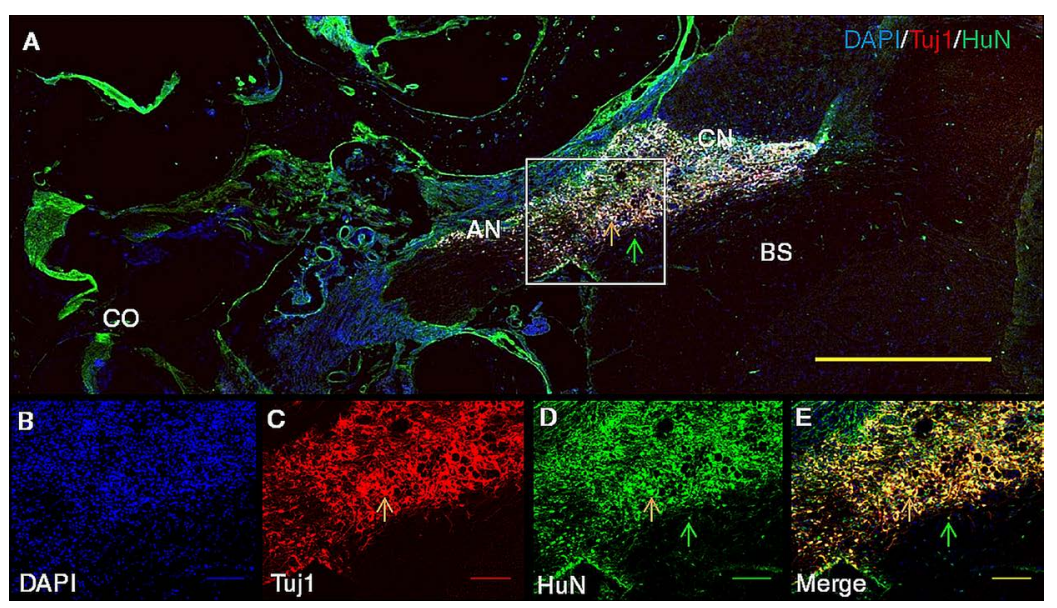

Figure 5. Representative macroscopic histological images of survival and migration of the regenerated structures towards $\mathrm{CN}$, 6 weeks post transplantation of HNPCs along with BDNF, ChABC and Bgel into the rat AN. Upper panels depict representative patterns of immunostaining for Tuj1 ir-positive HNPCs along the AN and CN (A, panoramic view, at 5× magnification with scale bar of $400 \mu \mathrm{m}$ (yellow) and lower panels depict representative patterns of immunostaining at $10 \times$ magnification with respective colored scale bars of $100 \mu \mathrm{m}$ for DAPI nuclear staining (B) (blue), Tuj1 neuronal staining (C) (red), HuN nuclear staining (D) (green), DAPI + Tuj1 + HuN co-expression (E) (yellow). Rectangle (white) indicates selected area of illustration. Arrows indicate that HNPCs are colabeled for Tuj1 and HuN (orange) and single labeled for HuN alone (green). Abbreviations: $\mathrm{AN}$, auditory nerve; $\mathrm{BS}$, brain stem; $\mathrm{CN}$, cochlear nucleus; $\mathrm{CO}$, cochlea.

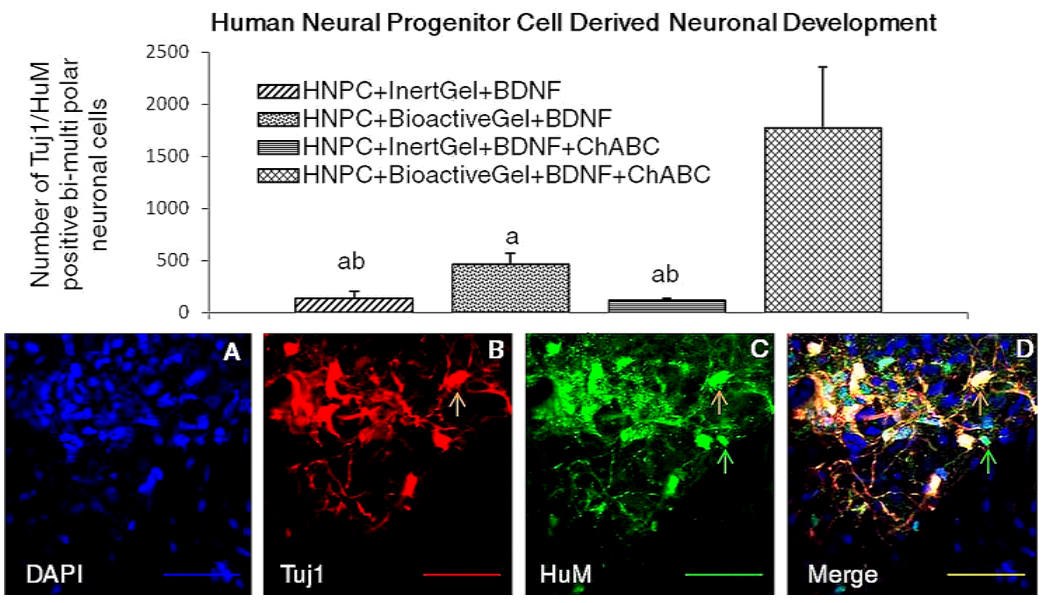

Figure 6. Effects of BDNF and ChABC treatment in the presence or absence of bioactive PA gel on the mean numbers of HNPC derived bi to multi-polar neuronal structures in characterized auditory loci in the female rat. $n=5$, Significant differences: ${ }^{a} P<0.05$, compared to HNPC + BioactiveGel + BDNF + ChABC; ${ }^{b} P<$ 0.05 , compared to HNPC + BioactiveGel + BDNF; ${ }^{c} P<0.05$, HNPC + InertGel + BDNF + ChABC. The lower panels depict representative patterns of immunostaining for Tuj1 ir-positive HNPCs showing axonal or dendritic mitochondrial transport at 20× magnification with respective colored scale bars of $50 \mu \mathrm{m}$ for DAPI nuclear staining (A) (blue), Tuj1 neuronal staining (B) (red), HuM mitochondrial staining (C) (green), DAPI + Tuj1 + HuM co-expression (D) (yellow) Arrows indicate that HNPCs are colabeled for Tuj1 and HuM (orange) and single labeled for HuM alone (green). Abbreviations: BDNF, brain derived neurotrophic factor; Bgel, bioactive peptide amphiphile gel; ChABC, chondroitinase ABC; HNPC, human neural progenitor cells; Igel, inert control peptide amphiphile gel. 
hair cells of the organ of Corti and supportive secretions like NT3 by other types of cells including SGN themselves [43]. These neurotrophic factors help the sensory SGNs to survive and maintain their functionality. The exogenous administration of these factors like BDNF, GDNF, NT-3 has shown to improve survival and development these neurons in vitro as well as when transplanted in vivo [44]. These surviving transplanted cells have shown to possess receptors for these growth factor ligands [45] [46]. BDNF has specifically shown to have positive effect on the survival of SGN and neurite growth as well [47] [48]. Also it has been shown that exogenous BDNF rescues SGN in vivo [49]. So it has been seen that various studies have tried to use BDNF as one of the key component additive to the cell replacement studies. In this study, exogenous BDNF is being used primarily for the purpose of maintaining survival and further development of the transplanted HNPCs. In previous studies, it has been demonstrated that the group with BDNF treatment showed a marked improvement in survival of the HNPCs, which suggests that BDNF is one of the key components that provides trophic support to these cells over a longer duration.

In the present study we also used a bioactive PA Bgel which is designed to present to the cells the neuritepromoting epitope IKVAV [34]. This carrier gel was mixed with BDNF/ChABC along with HNPCs and this combination was injected into the AN. We tried to make use of the nanofiber network of this gel to incorporate cells and the modulating agents together. In this combination, the PA gel might have provided a unique advantage of releasing these incorporated modulators in a proportionate manner and the gel might have also worked as a scaffold to these cells providing a unique nourishing environment. Since the gel possesses a property of promoting neural differentiation, this scaffolding might have assisted in the development of HNPCs. Also since the gel has been shown to inhibit glial scar formation [50], this unique property along with its assistance in differentiation may have certainly contributed to the longer survival of the HNPCs and development towards showing neuronal phenotype resembling cellular characteristic. Although we are speculating a beneficial role of the PA bioactive gel in this study, we do recognize that further examination at a molecular level is needed in future. The survival rate of HNPCs has been moderate in comparison to the survival of Mouse Tau-GFP cells in previous studies but this study was done over a longer duration of six weeks of survival time and that might have had an effect on the cell number. Also, the survival and differentiation was mostly seen towards the CN as compared to the peripheral cochlear region which suggested a clear attraction of these cells towards the CNS that has been already shown in vitro [27]. But the non-migration of these cells towards the cochlear peripheral region after longer survival time cannot be overlooked and needs further investigation.

Since the implanted cells travelled across the AN and reached the cochlear nucleus, the data also speculates that the HNPCs travelled along the AN passing through the TZ. We have previously seen that following cell transplantation into AN, the migration of the cells as well as outgrowth of neuronal fibers have been hindered by the presence of TZ separating PNS from the CNS [29]. This hindrance is only seen in a PNS to CNS direction and not vise versa [36]. In this study we used ChABC, an enzyme that possesses an ability to break chondroitin sulphate glycosaminoglycan (GAG) chains of $\mathrm{TZ}$ and makes it permeable to allow migration or axonal growth towards the CNS. Also in response to any scar formation, as is the case in AN damage, there is an upregulation of Chondroitin sulphate proteoglycan and that inhibits axonal sprouting and further development. By using ChABC, we might have indirectly reduced the production of these proteoglycans and thereby their detrimental effects on the neuronal regeneration. That is why we could observe a great deal of axonal sprouting and elongation from the surviving HNPCs. Previously it has been observed that the mouse Tau-GFP ESCs when transplanted with ChABC showed a great deal of migration throughout the AN and into the brain stem. This is seen after a 3 weeks survival time. Similar approach has shown that the ChABC substantially facilitated the migration of HNPCs towards the CN and formation of new axons directing towards CNS over a period of 6 weeks.

Tuj1 and HuN co-expression was seen in numerous surviving cells and they showed axonal or dendritic sprouting forming bi to multipolar neuronal phenotype suggesting these neuronal cells can occur within the polysynaptic circuitry that links peripheral and central sensory mechanism that regulate hearing function. Further work is needed to characterize these neurochemical phenotypes and neuroanatomical connectivity of these formed neuronal structures. Our findings that the transplanted HNPCs survived after 6 weeks and that the ChABC promoted axonal growth and sprouting might suggest that the AN neuronal damage mechanism process has been abated and compensated. The current evidence that there are formation new axonal and dendritic processes supports the view that ChABC has an inhibitory effect on the upregulation the injury related proteoglycans. Also, the BS oriented migration and elongation of neurite growth supports a likelihood of modulatory trophic support and attraction from BS. Although we added exogenous trophic factors for the survival of the 
HNPCs, we cannot rule out the role of intrinsic physiological trophic support provided by the residual amount of SGNs and intact hair cells that were present in the near vicinity. Also, the close proximity of the migrated HNPCs to the CN may suggest an abundance of trophic factors availability at the BS area to support the survival and development of the HNPCs, though a further investigative role for this BS bound attraction phenomenon is needed to assess the hearing function.

The current data also show that a moderate number of human nuclei marker positive cells do not express Tuj1. Such results do not preclude the possibility of these cells to form neuronal phenotypes in future. Also the Tuj1 immuno-positive cells confirm a neuronal phenotype and it can be seen that there is mitochondrial movement along the axons and dendrites of these phenotypes. It is important to note that the axonal and dendritic development was of substantial level in ChABC treated HNPCs.

\section{Conclusion}

In summary, the present study demonstrates that the HNPCs when transplanted along with exogenous biomimetic modulatory factors like BDNF, PA bioactive gel and ChABC show appreciable survival, migration and differentiation after 6 weeks of survival time. The survival appeared to be along the AN migrating into the CN area, which suggests widespread migration of these cells. The differentiation of these cells into neuronal phenotypes having axonal and dendritic growth suggests a role for ChABC in cell based transplantation strategy. Although the development of these neuronal structures into auditory functional cell types has not been established yet, further studies will help explore the phenotypic characteristics of these neuronal structures. Moreover, the formation of CNS related sensory structures could not be ruled out as well, since these cells show a prominent dendritic development with mitochondrial transport along them. So it will be interesting to study the sensory cell profiles of these developing cells into a functional auditory output. Overall in a broader perspective, this regenerative transplantation study shows promising results endorsing a clinically transferable futuristic role for biomaterial consisted neutrophic factor application in cell based treatment of sensorineural hearing loss along with cochlear implants.

\section{Acknowledgements}

P. Olivius: This work was supported by The Swedish Research Council no. 2008-2822, Marianne and Marcus Wallenbergs Foundation, Petrus and Augusta Hedlunds Foundation, The Swedish Association of Hard of Hearing People, Acta Otolaryngologica’s Foundation, The Foundation Tysta Skolan, Ollie and Elof Ericssons Foundation for Medical Research and Karolinska Institutet Foundations. U. Englund-Johansson: This work was supported by the Medical faculty and Lund University. The funders had no role in the study design, data collection and analysis, decision to publish, or preparation of the manuscript.

\section{References}

[1] Nadol, J. (1997) Patterns of Neural Degeneration in the Human Cochlea and Auditory Nerve: Implications for Cochlear Implantation. Otolaryngology_-Head and Neck Surgery, 117, 220-228. http://dx.doi.org/10.1016/S0194-5998(97)70178-5

[2] Johnsson, L.G., Felix, H., Gleeson, M. and Pollak, A. (1990) Observations on the Pattern of Sensorineural Degeneration in the Human Cochlea. Acta otolaryngologica Supplementum, 470, 88-95.

[3] Moser, T., Predoehl, F. and Starr, A. (2013) Review of Hair Cell Synapse Defects in Sensorineural Hearing Impairment. Otology \& Neurotology, 34, 995-1004. http://dx.doi.org/10.1097/MAO.0b013e3182814d4a

[4] Spelman, F. (1999) The Past, Present, and Future of Cochlear Prostheses. IEEE Engineering in Medicine and Biology Magazine, 18, 27-33. http://dx.doi.org/10.1109/51.765186

[5] McCreery, D. (2008) Cochlear Nucleus Auditory Prostheses. Hearing Research, 242, 64-73. http://dx.doi.org/10.1016/j.heares.2007.11.014

[6] Hildebrand, M.S., Newton, S.S., Gubbels, S.P., Sheffield, A.M., Kochhar, A., De Silva, M.G., Dahl, H.H., Rose, S.D., Hehlke, M.A. and Smith, R.J. (2008) Advances in Molecular and Cellular Therapies for Hearing Loss. Molecular Therapy: The Journal of the American Society of Gene Therapy, 16, 224-236. http://dx.doi.org/10.1038/sj.mt.6300351

[7] Møller, A. (2001) Neurophysiologic Basis for Cochlear and Auditory Brainstem Implants. American Journal of Audiology, 10, 68-77. http://dx.doi.org/10.1044/1059-0889(2001/012)

[8] Jia, H., Wang, J., François, F., Uziel, A., Puel, J.L. and Venail, F. (2013) Molecular and Cellular Mechanisms of Loss 
of Residual Hearing after Cochlear Implantation. The Annals of Otology, Rhinology, and Laryngology, 122, 33-39.

[9] Zimmermann, C., Burgess, B. and Nadol, J. (1995) Patterns of Degeneration in the Human Cochlear Nerve. Hearing Research, 90, 192-201. http://dx.doi.org/10.1016/0378-5955(95)00165-1

[10] Zhang, P.Z., He, Y., Jiang, X.W., Chen, F.Q., Chen, Y., Shi, L., Chen, J., Chen, X., Li, X., Xue, T., Wang, Y., Mi, W.J. and Qiu, J.H. (2013) Stem Cell Transplantation via the Cochlear Lateral Wall for Replacement of Degenerated Spiral Ganglion Neurons. Hearing Research, 298, 1-9. http://dx.doi.org/10.1016/j.heares.2013.01.022

[11] Hu, Z.Q., Ulfendahl, M., Prieskorn, D.M., Olivius, P. and Miller, J.M. (2009) Functional Evaluation of a Cell Replacement Therapy in the Inner Ear. Otology \& Neurotology, 30, 551-558. http://dx.doi.org/10.1097/MAO.0b013e31819fe70a

[12] Jongkamonwiwat, N., Zine, A. and Rivolta, M. (2010) Stem Cell Based Therapy in the Inner Ear: Appropriate Donor Cell Types and Routes for Transplantation. Current Drug Targets, 11, 888-897. http://dx.doi.org/10.2174/138945010791320836

[13] Kesser, B. and Lalwani, A. (2009) Gene Therapy and Stem Cell Transplantation: Strategies for Hearing Restoration. Advances in Oto-Rhino-Laryngology, 66, 64-86. http://dx.doi.org/10.1159/000218208

[14] Lefèbvre, P., Malgrange, M. and Moonen, M. (2008) Regeneration of Hair Cells and Auditory Neurons in the Ear. Bulletin et Mémoires de l’Académie Royale de Médecine de Belgique, 163, 391-396.

[15] Chen, J. and Streit, A. (2013) Induction of the Inner Ear: Stepwise Specification of Otic Fate from Multipotent Progenitors. Hearing Research, 297, 3-12. http://dx.doi.org/10.1016/j.heares.2012.11.018

[16] Alonso, M.B.D., Feijoo-Redondo, A., de Felipe, M.C., Carnicero, E., Garcia, A.S., Garcia-Sancho, J., Rivolta, M.N., Giraldez, F. and Schimmang, T. (2012) Generation of Inner Ear Sensory Cells from Bone Marrow-Derived Human Mesenchymal Stem Cells. Regenerative Medicine, 7, 769-783. http://dx.doi.org/10.2217/rme.12.65

[17] Martinez-Monedero, R. and Edge, A. (2007) Stem Cells for the Replacement of Inner Ear Neurons and Hair Cells. The International Journal of Developmental Biology, 51, 655-661. http://dx.doi.org/10.1387/ijdb.072372rm

[18] Pettingill, L.N., Richardson, R.T., Wise, A.K., O’Leary, S.J. and Shepherd, R.K. (2007) Neurotrophic Factors and Neural Prostheses: Potential Clinical Applications Based upon Findings in the Auditory System. IEEE Transactions on Bio-Medical Engineering, 54, 1138-1148. http://dx.doi.org/10.1109/TBME.2007.895375

[19] Ferretti, P. (2011) Is There a Relationship between Adult Neurogenesis and Neuron Generation Following Injury across Evolution? The European Journal of Neuroscience, 34, 951-962. http://dx.doi.org/10.1111/j.1460-9568.2011.07833.x

[20] Löwenheim, H., Waldhaus, J., Hirt, B., Sandke, S. and Müller, M. (2008) Regenerative Medicine in the Treatment of Sensorineural Hearing Loss. HNO, 56, 288-300. http://dx.doi.org/10.1007/s00106-008-1689-y

[21] Vlastarakos, P.V., Nikolopoulos, T.P., Tavoulari, E., Kiprouli, C. and Ferekidis, E. (2008) Novel Approaches to Treating Sensorineural Hearing Loss. Auditory Genetics and Necessary Factors for Stem Cell Transplant. Medical Science Monitor : International Medical Journal of Experimental and Clinical Research, 14, RA114-RA125.

[22] Ramsden, R. (2002) Cochlear Implants and Brain Stem Implants. British Medical Bulletin, 63, 183-193. http://dx.doi.org/10.1093/bmb/63.1.183

[23] Dillon, M.T., Buss, E., Adunka, M.C., King, E.R., Pillsbery 3rd, H.C., Anduka, O.F. and Buchman, C.A. (2013) LongTerm Speech Perception in Elderly Cochlear Implant Users. JAMA Otolaryngology, Head \& Neck Surgery, 139, 279283.

[24] Barker, R. (2012) Stem Cells and Neurodegenerative Diseases: Where Is It All Going? Regenerative Medicine, 7, 2631. http://dx.doi.org/10.2217/rme.12.64

[25] Incesulu, A. and Nadol, J.B. (1998) Correlation of Acoustic Threshold Measures and Spiral Ganglion Cell Survival in Severe to Profound Sensorineural Hearing Loss: Implications for Cochlear Implantation. The Annals of Otology, Rhinology, and Laryngology, 107, 906-911.

[26] Goffi-Gomez, M.V., Magalhães, A., Brito Neto, R., Tsuji, R.K., Gomes, M. and Bento, R.F. (2012) Auditory Brainstem Implant Outcomes and MAP Parameters: Report of Experiences in Adults and Children. International Journal of Pediatric Otorhinolaryngology, 76, 257-264. http://dx.doi.org/10.1016/j.ijporl.2011.11.016

[27] Novozhilova, E., Olivius, P., Siratirakun, P., Lundberg, C. and Englund-Johansson, U. (2013) Neuronal Differentiation and Extensive Migration of Human Neural Precursor Cells following Co-Culture with Rat Auditory Brainstem Slices. PLoS ONE, 8, e57301. http://dx.doi.org/10.1371/journal.pone.0057301

[28] Glavaski-Joksimovic, A., Thonabulsombat, C., Wendt, M., Eriksson, M., Ma, H. and Olivius, P. (2009) Morphological Differentiation of Tau-Green Fluorescent Protein Embryonic Stem Cells into Neurons after Co-Culture with Auditory Brain Stem Slices. Neuroscience, 162, 472-481. http://dx.doi.org/10.1016/j.neuroscience.2009.04.070

[29] Palmgren, B., Jiao, Y., Novozhilova, E., Stupp, S.I. and Olivius, P. (2012) Survival, Migration and Differentiation of 
Mouse Tau-GFP Embryonic Stem Cells Transplanted into the Rat Auditory Nerve. Experimental Neurology, 235, 599609. http://dx.doi.org/10.1016/j.expneurol.2012.03.014

[30] Rask-Andersen, H., Boström, M., Gerdin, B., Kinnefors, A., Nyberg, G., Engstrand, T., Miller, J.M. and Lindholm, D. (2005) Regeneration of Human Auditory Nerve. In Vitro/in Video Demonstration of Neural Progenitor Cells in Adult Human and Guinea Pig Spiral Ganglion. Hearing Research, 203, 180-191. http://dx.doi.org/10.1016/j.heares.2004.12.005

[31] Pyle, A., Lock, L. and Donovan, P. (2006) Neurotrophins Mediate Human Embryonic Stem Cell Survival. Nature Biotechnology, 24, 344-350. http://dx.doi.org/10.1038/nbt1189

[32] Armstrong, R.J., Watts, C., Svendsen, C.N., Dunnett, S.B. and Rosser, A.E. (2000) Survival, Neuronal Differentiation, and Fiber Outgrowth of Propagated Human Neural Precursor Grafts in an Animal Model of Huntington's Disease. Cell Transplantation, 9, 55-64.

[33] Palmgren, B., Jin, Z., Ma, H., Jiao, Y. and Olivius, P. (2010) Beta-Bungarotoxin Application to the Round Window: An in Vivo Deafferentation Model of the Inner Ear. Hearing Research, 265, 70-76. http://dx.doi.org/10.1016/j.heares.2010.02.009

[34] Silva, G.A., Czeisler, C., Niece, K.L., Beniash, E., Harrington, D.A., Kessler, J.A. and Stupp, S.I. (2004) Selective Differentiation of Neural Progenitor Cells by High-Epitope Density Nanofibers. Science, 303, 1352-1355. http://dx.doi.org/10.1126/science.1093783

[35] Toesca, A. (1996) Central and Peripheral Myelin in the Rat Cochlear and Vestibular Nerves. Neuroscience Letters, 221, 21-24. http://dx.doi.org/10.1016/S0304-3940(96)13273-0

[36] Fraher, J. (2000) The Transitional Zone and CNS Regeneration. Journal of Anatomy, 196, 137-158.

[37] Grimpe, B., Pressman, Y., Lupa, M.D., Horn, K.P., Bunge, M.B. and Silver, J. (2005) The Role of Proteoglycans in Schwann Cell/Astrocyte Interactions and in Regeneration Failure at PNS/CNS Interfaces. Molecular and Cellular Neurosciences, 28, 18-29. http://dx.doi.org/10.1016/j.mcn.2004.06.010

[38] Zuo, J., Neubauer, D., Graham, J., Krekoski, C.A., Ferguson, T.A. and Muir, D. (2002) Regeneration of Axons after Nerve Transection Repair Is Enhanced by Degradation of Chondroitin Sulfate Proteoglycan. Experimental Neurology, 176, 221-228. http://dx.doi.org/10.1006/exnr.2002.7922

[39] Suzuki, T., Akimoto, M., Imai, H., Ueda, Y., Mandai, M., Yoshimura, N., Swaroop, A. and, Takahashi, M. (2007) Chondroitinase ABC Treatment Enhances Synaptogenesis between Transplant and Host Neurons in Model of Retinal Degeneration. Cell Transplantation, 16, 493-503.

[40] Englund, U., Ericson, C., Rosenblad, C., Mandel, R.J., Tono, D., Wictorin, K. and Lundberg, C. (2000) The Use of a Recombinant Lentiviral Vector for ex Vivo Gene Transfer into the Rat CNS. Neuroreport, 11, 3973-3977. http://dx.doi.org/10.1097/00001756-200012180-00014

[41] Carpenter, M.K., Cui, X., Hu, Z.Y., Jackson, J., Sherman, S., Seiger, Å. and Wahlberg, L.U. (1999) In Vitro Expansion of a Multipotent Population of Human Neural Progenitor Cells. Experimental Neurology, 158, 265-278. http://dx.doi.org/10.1006/exnr.1999.7098

[42] Roehm, P. and Hansen, M. (2005) Strategies to Preserve or Regenerate Spiral Ganglion Neurons. Current Opinion in Otolaryngology and Head and Neck Surgery, 13, 294-300. http://dx.doi.org/10.1097/01.moo.0000180919.68812.b9

[43] Wheeler, E.F., Bothwell, M., Schecterson, L.C. and Von Bartheld, C.S. (1994) Expression of BDNF and NT-3 mRNA in Hair Cells of the Organ of Corti: Quantitative Analysis in Developing Rats. Hearing Research, 73, 46-56. http://dx.doi.org/10.1016/0378-5955(94)90281-X

[44] Hess, D. and Borlongan, C. (2008) Stem Cells and Neurological Diseases. Cell Proliferation, 241, 94-114.

[45] Wiechers, B., Gestwa, G., Mack, A., Carroll, P., Zenner, H.P. and Knipper, M. (1999) A Changing Pattern of BrainDerived Neurotrophic Factor Expression Correlates with the Rearrangement of Fibers during Cochlear Development of Rats and Mice. The Journal of Neuroscience, 19, 3033-3042.

[46] Bergami, M., Vignoli, B., Motori, E., Pifferi, S., Zuccaro, E., Menini, A. and Canossa, M. (2013) TrkB Signaling Directs the Incorporation of Newly Generated Periglomerular Cells in the Adult Olfactory Bulb. Journal of Neuroscience, 33, 11464-11478. http://dx.doi.org/10.1523/JNEUROSCI.4812-12.2013

[47] Kondo, K., Pak, K., Chavez, E., Mullen, L., Eunteneuer, S. and Ryan, A.F. (2013) Changes in Responsiveness of Rat Spiral Ganglion Neurons to Neurotrophins across Age: Differential Regulation of Survival and Neuritogenesis. The International Journal of Neuroscience, 123, 465-475. http://dx.doi.org/10.3109/00207454.2013.764497

[48] Jin, Y., Kondo, K., Ushio, M., Kaga, K., Ryan, A.F. and Yamasoba, T. (2013) Developmental Changes in the Responsiveness of Rat Spiral Ganglion Neurons to Neurotrophic Factors in Dissociated Culture: Differential Responses for Survival, Neuritogenesis and Neuronal Morphology. Cell and Tissue Research, 351, 15-27.

http://dx.doi.org/10.1007/s00441-012-1526-1 
[49] Landry, T.G., Wise, A.K., Shepherd, R.K. and Fallon, J.B. (2011) Spiral Ganglion Neuron Survival and Function in the Deafened Cochlea Following Chronic Neurotrophic Treatment. Hearing Research, 282, 303-313. http://dx.doi.org/10.1016/j.heares.2011.06.007

[50] Tysseling, V.M., Sahni, V., Pashuck, E.T., Birch, D., Hebert, A., Czeisler, C., Stupp, S.I. and Kessler, J.A. (2010) SelfAssembling Peptide Amphiphile Promotes Plasticity of Serotonergic Fibers Following Spinal Cord Injury. Journal of Neuroscience Research, 88, 3161-3170. http://dx.doi.org/10.1002/jnr.22472 